\title{
Rischio biologico connesso al confezionamento, trasporto ed accettazione del campione
}

\author{
Marina Crovatto*, Paola Gualdi**
}

* S.O.S. Immunologia Clinica e Virologia, Dipartimento di Medicina di Laboratorio, Azienda Ospedaliera Santa Maria degli Angeli, Pordenone

** Laboratorio Analisi Chimicocliniche e Microbiologia , Ospedale S. Maria del Carmine Rovereto(TN)

\section{INTRODUZIONE}

Le infezioni occupazionali sono un problema quotidianamente presente per il laboratorista: il rischio di contrarle è reale, anche se difficile da quantificare data la mancanza di sistemi di sorveglianza adeguati sia a livello nazionale che internazionale. I primi dati significativi a tal proposito sono riportati nel noto lavoro di Pike et al. del 1976, secondo il quale su 3921 casi di infezioni contratte in laboratorio, 2465 si erano verificate negli USA e 164 erano risultate fatali. Solo il $64 \%$ dei casi era reperibile in letteratura, solo il $18 \%$ era correlabile con incidenti documentati, il $42 \%$ era dovuto a batteri, il $27 \%$ a virus, il $15 \%$ a Rickettsie, il $9 \%$ a funghi, il 3\% a Chlamydia e un ulteriore $3 \%$ a parassiti. Da allora il problema è sempre stato oggetto di maggior attenzione, accentuata anche dalla comparsa di HIV e le segnalazioni hanno subito un rapido incremento $(5,9,11,15,16,18)$.

Per affrontare un argomento di questo tipo è comunque doveroso richiamare alcuni concetti:

- pericolo: proprietà o qualità intrinseca di una determinata entità avente il potenziale di causare danni

- rischio: probabilità che, in presenza di un pericolo, si manifesti un evento indesiderato comportante esposizione

- rischio di esposizione ad agenti biologici: rischio di venire a contatto mediante inoculazione, ingestione (contaminazione delle superfici $\rightarrow$ mani $\rightarrow$ mucose bocca) contatto cutaneo o mucoso diretto o indiretto, inalazione di aerosol o particelle trasportate per via aerea con qualsiasi microorganismo o coltura cellulare o endoparassita umano agente di infezione, allergia, intossicazione nell'uomo.

Il pericolo è rappresentato dagli agenti biologici a cui l'operatore può essere esposto durante l'espletamento del proprio lavoro e che vengono suddivisi in 4 gruppi, in base al grado di pericolosità:

Agente biologico di gruppo 1: agente che presenta poche probabilità di malattia in soggetti umani;

Agente biologico di gruppo 2: agente che può causare malattia in soggetti umani e costituire un rischio per i lavoratori, è poco probabile che si propaghi nella comunità, sono di norma disponibili efficaci misure profilattiche o terapeutiche;

Agente biologico di gruppo 3: agente che può causare malattie gravi in soggetti umani e costituisce un serio rischio per i lavoratori; può propagarsi nella comunità, ma di norma sono disponibili efficaci misure profilattiche o terapeutiche;

Agente biologico di gruppo 4: agente che può provocare malattie gravi in soggetti umani e può presentare un elevato rischio di propagazione nella comunità: non sono disponibili, di norma, efficaci misure profilattiche o terapeutiche Il gruppo di maggiore pericolosità per gli operatori sanitari è rappresentato prevalentemente dal gruppo 3 (es.HBV, HCV, HIV, M.tuberculosis ecc...), mentre gli agenti del gruppo 4 , al momento, sono confinati in aree geografiche limitate quali ad esempio alcune zone dell'Africa.

Manipolando campioni biologici, siamo costantemente a rischio di esposizione agli agenti biologici per cui conoscere le loro caratteristiche, il loro habitat, la capacità di sopravvivenza nell'ambiente, come si comportano quando il nostro organismo viene accidentalmente a contatto con essi, diventa essenziale per mettere in atto tutti gli accorgimenti e le procedure necessarie per la tutela della nostra salute.

\section{SOPRAVVIVENZA DI ALCUNI AGENTI BIOLOGICI NELL'AMBIENTE E/O SULL'EPIDERMIDE}

Il comportamento degli agenti biologici al di fuori dell'organismo è estremamente vario e dipendente dalle loro caratteristiche. I cocchi Gram positivi e tra i bacilli Gram negativi l'Acinetobacter calcoaceticus resistono bene nell'ambiente: in condizioni di essiccamento rimangono vitali oltre 25 giorni I bacilli Gram negativi non $A$. calcoaceticus perdono invece rapidamente vitalità (in circa 7 ore); la presenza di proteine aumenta notevolmente la capacità di sopravvivenza dei batteri in tali condizioni. Secondo alcuni Autori in condizioni di essiccamento ed in presenza di materiale organico, Staphylococcus aureus perde vitalità tra 3 e 6 mesi mentre Escherichia coli e 
Pseudomonas aeruginosa sopravvivono più a lungo. In assenza di materiale organico la sopravvivenza non supera le quattro settimane per nessuno dei microrganismi citati $(14,16)$. Secondo rilievi recenti il Virus Respiratorio Sinciziale (RSV) è in grado di resistere sulla pelle e sulle superfici porose (vestiti, carta) fino a 30 minuti, sulle superfici non porose (es. guanti) fino a 6 ore. I virus parainfluenzali persistono sulle superfici porose fino a 4 ore, sulle non porose (es. acciaio) fino a 10 ore, mentre dopo 10 ' di contatto, sui polpastrelli è rilevabile solo il 5\% dei virus con cui si è avuto il contatto. L'essiccamento determina l'inattivazione del $90-99 \%$ di HIV in 1-2 ore, però, utilizzando concentrazioni 100.000 volte superiori a quelle riscontrate nel sangue di persone infette, è stato rilevato vitale dopo 1-3 giorni dall'essiccamento. In soluzioni acquose HIV può persistere, a temperatura ambiente, per 2 settimane, sopravvive 7-10 giorni a $4^{\circ} \mathrm{C}$, fino a 6 giorni in cadavere $\left(\right.$ a $\left.6^{\circ} \mathrm{C}\right)(16)$. La sopravvivenza di HIV nelle siringhe, dipende dalla temperatura, quantità di sangue, carica viale, tempo di permanenza a temperatura ambiente e ceppo: vengono riportati range variabili da 28 a 42 giorni. HBV presenta caratteristiche di resistenza alle condizioni ambientali, superiori ai precedenti; riesce infatti a rimanere vitale sulle superfici, dopo essiccamento, fino a 1 settimana e probabilmente anche più a lungo. Il Coronavirus responsabile della SARS (SARS-CoV) è in grado di sopravvivere nelle feci e nelle urine, a temperatura ambiente, per 1-2 giorni; nelle feci diarroiche ( $\mathrm{pH}$ elevato) fino a 4 giorni. Nei surnatanti delle colture cellulari dopo 21 giorni a $4^{\circ} \mathrm{C}$ e $-80^{\circ} \mathrm{C}$ è stata rilevata una riduzione minima della sua concentrazione che invece calava di $1 \log$ quando i surnatanti venivano conservati a temperatura ambiente. È sensibile al calore: il trattamento a $56^{\circ} \mathrm{C}$ inattiva 10.000 Unità di virus in 15 minuti. In una recente sperimentazione è stata saggiata la sopravvivenza di SARS$\mathrm{CoV}$ su vari tipi di superfici mediante contaminazione, a temperatura ambiente, delle stesse con il ceppo virale in PBS o in feci sterilizzate (per valutare l'effetto protettivo delle sostanze organiche) (WHO). I risultati sono i seguenti:

\begin{tabular}{lll}
\hline & PBS & Feci \\
\hline Pareti & $24 \mathrm{~h}$ & $36 \mathrm{~h}$ \\
\hline Superfici di plastica & $36 \mathrm{~h}$ & $72 \mathrm{~h}$ \\
\hline Superfici di formica & $36 \mathrm{~h}$ & $36 \mathrm{~h}$ \\
\hline Acciaio & $36 \mathrm{~h}$ & $72 \mathrm{~h}$ \\
\hline Legno & $12 \mathrm{~h}$ & $24 \mathrm{~h}$ \\
\hline Tessuto di cotone & $12 \mathrm{~h}$ & $24 \mathrm{~h}$ \\
\hline Vetro & $72 \mathrm{~h}$ & $96 \mathrm{~h}$ \\
\hline Copertine di raccoglitori & $24 \mathrm{~h}$ & $36 \mathrm{~h}$ \\
\hline
\end{tabular}

L'analisi del ceppo CoV-P9 isolato da tampone faringeo di un probabile caso di SARS ha sostanzialmente confermato le buone capacità di sopravvivenza del virus nell'ambiente e la sua sensibilità alla temperatura ed agli UV.

Il Mycobacterium tuberculosis riesce a sopravvivere sui vetrini fissati al calore (non colorati) per almeno 7 giorni, mentre il Mycobacterium avium complex dopo essiccamento perde rapidamente vitalità (emivita di 2,3 giorni). Il Mycobacterium bovis sopravvive per più di due mesi dopo essiccamento. I Micobatteri sono resistenti in ambiente buio e sopravvivono per mesi in condizioni di temperatura ed umidità ottimali, mentre vengono inattivati dagli $U V$, alla luce del sole $(1,2,3,4,7$, $10,13,14,17)$. Al di là dei singoli comportamenti è chiaro che il livello di attenzione per evitare fuoriuscite accidentali di campioni biologici ed il contatto con gli stessi, deve essere sempre ai massimi livelli e che le procedure per operare "in sicurezza" devono essere accuratamente seguite: è il mezzo più sicuro ed efficace per evitare le infezioni occupazionali.

\section{PRINCIPALI AGENTI DI INFEZIONI OCCUPAZIONALI}

Mycobacterium tuberculosis: è presente nell'escreato, liquido di lavaggio bronchiale, liquido di lavaggio gastrico, liquor, urine, materiale da lesioni caseose. La trasmissione agli operatori sanitari avviene per via aerea. Generalmente la dose infettante è di 1-10 Micobatteri trasportati in 1-3 droplet nucleus.

Virus dell'epatite $\boldsymbol{B}(\boldsymbol{H B V})$ : la concentrazione di HBV a livello ematico generalmente può variare da pochi virioni a $10^{9}-10^{11}$ virioni $/ \mathrm{ml}$. Il virus può essere presente anche nella saliva, sperma, secrezioni vaginali. Nella saliva e nello sperma la concentrazione è 1.000-10.000 volte inferiore rispetto al sangue. Può essere presente in concentrazioni molto basse anche nelle urine. La modalità principale di trasmissione per gli operatori sanitari è la puntura accidentale. La trasmissione dipende dalla concentrazione del virus, dal volume di materiale infettante con cui il soggetto viene a contatto, dal tipo di esposizione (parenterale o mucosa).

La quantità minima di sangue con la quale si deve venire a contatto per avere la probabilità di contrarre infezione secondo l'Organizzazione Mondiale della Sanità (WHO) è di $0,04 \mu \mathrm{l}$ (in caso di puntura accidentale con ago da 22, la quantità inoculata è circa $1 \mathrm{ul}$ che può contenere fino a 100 dosi infettanti di HBV). Il rischio di trasmissione è del $30 \%$ se il paziente fonte è $\mathrm{HBeAg}$ positivo, inferiore al $6 \%$ se il paziente fonte è $\mathrm{HBeAg}$ negativo. Secondo i dati del CDC 
1000 operatori sanitari hanno contratto l'infezione da HBV nel 1994, in USA, con una riduzione del $90 \%$ rispetto al 1987 (risultati vaccinazioni ed azioni preventive) e nel corso dell'ultima decade 100-200 operatori sono morti per le conseguenze legate all'infezione occupazionale $(5,6)$.

Virus dell'epatite $\boldsymbol{C}(\boldsymbol{H C V})$ : la concentrazione del virus nel sangue varia da pochi virioni a $10^{7}$ $\mathrm{UI} / \mathrm{ml}$. Il virus può essere presente anche nella saliva e nello sperma. Non è stato rilevato nelle urine, feci o secrezioni vaginali. La trasmissione dipende dalla concentrazione del virus, dalla quantità di inoculo, dal tipo di esposizione. Non vi sono dati sufficienti per stabilire qual è il valore soglia di concentrazione necessaria per avere la probabilità di trasmissione dell'infezione. La modalità di trasmissione principale per gli operatori sanitari è la puntura accidentale. Il rischio di trasmissione è dell' $1,8 \%$ (range $0-7 \%$ ) se il paziente fonte è anti-HCV positivo, del $10 \%$ se il paziente fonte è HCV RNA positivo. Non vi sono segnalazioni di casi di infezione dopo esposizione delle mucose o cute non integra. Sono comunque riportati due casi di trasmissione di $\mathrm{HCV}$ dovuta a schizzo di sangue sulla congiuntiva (5). Secondo i dati del CDC il 2-4\% del numero annuale (180.000 nel 1984, 28.000 nel 1995) di nuove infezioni da HCV che si hanno in USA si verifica in operatori sanitari esposti a sangue durante l'attività lavorativa.

Virus dell'immunodeficienza umana (HIV): la concentrazione di HIV nel sangue varia da pochi virioni a $10^{7}-10^{8}$ virioni $/ \mathrm{ml}$. Può essere presente anche nella saliva, sperma, latte materno, tessuti, liquido amniotico, liquido cerebro-spinale, liquido sinoviale, liquido peritoneale, liquido pleurico, liquido pericardico. Generalmente è assente nelle feci, secrezioni nasali, escreato, sudore, lacrime, urine, vomito, a meno che non contengano sangue in quantità visibile. La puntura accidentale rappresenta la modalità di trasmissione principale per gli operatori sanitari. La trasmissione dipende dalla concentrazione del virus, dalla quantità di inoculo, dal tipo di esposizione, dalle caratteristiche del paziente fonte. È stato infatti dimostrato che la quantità di virus presente nel sangue di soggetti con AIDS conclamato è significativamente più alta che nei soggetti con infezione asintomatica. La quantità minima di sangue con la quale si deve venire a contatto per avere la probabilità di contrarre infezione, secondo l'Organizzazione Mondiale della Sanità (WHO) è 100 ul. Sulla base delle valutazioni fino ad ora fatte, il rischio di contrarre infezione è dello $0,3 \%$ dopo esposizione parenterale, dello $0,09 \%$ dopo esposizione delle mucose. Non sono state fino ad ora dimostrate sieroconversioni in seguito ad esposizione della cute integra.

Non ci sono dati sufficienti per quantificare il rischio di trasmissione dopo esposizione a liquidi biologici potenzialmente infetti, diversi dal sangue, tuttavia, secondo uno studio condotto su 559 partecipanti che avevano riportato esposizione cutanea a sangue, escreato, urine, feci o altri liquidi corporei di pazienti presumibilmente infetti da HIV, nessuno si è infettato. Non ci sono attualmente prove che HIV venga trasmesso per via aerea. L'assenza di trasmissione per via aerea è indirettamente dimostrata dall'assenza di infezione in 1201 soggetti che hanno partecipato a procedure su pazienti infetti o con AIDS conclamato senza mai utilizzare protezioni per prevenire l'inalazione di aerosol (5).

Allo stato attuale delle conoscenze, in Italia sono state documentate 5 infezioni occupazionali da HIV, in letteratura sono stati descritti circa 100 casi di infezione occupazionale da HIV documentati con la sieroconversione o la tipizzazione biomolecolare del virus. Le categorie professionali che hanno contratto l'infezioni sono: infermieri, laboratoristi, medici, ausiliari, dentisti.

Altri agenti riportati in letteratura quali responsabili di casi di infezioni occupazionali (con frequenza inferiore rispetto ai precedenti):

- Batteri: Brucella spp., Burholderia (Pseudomonas) pseudomallei, Chlamydia spp., Francisella tularensis, Leptospira spp., Salmonella spp., Shigella spp. Raramente responsabili: Vibrio spp., Campylobacter spp., Escherichia coli, Treponema pallidum.

- Rickettsie: Coxiella burneti

- Virus: Herpesvirus simiae (B virus), Virus della coriomeningite linfocitaria, Virus della stomatite vescicolare: prevalentemente in operatori di laboratori di ricerca. Arenavirus e Filovirus (al momento presenti prevalentemente in alcune zone dell'Africa) per i quali viene comunque richiesto il livello di sicurezza 4 (BSL4), TBE, Hantavirus, Virus dell'influenza, RSV, Enterovirus, Virus della Parotite, Rosolia e Morbillo, HSV

- Miceti: Criptococcus neoformans, Blastomyces dermatitis, Coccidioides immitis, Histoplasma capsulatum

- Parassiti: Toxoplasma gondii, Plasmodium spp., Leishmania spp., Tripanosoma spp. (16)

CONFEZIONAMENTO, TRASPORTO ED ACCETTAZIONE DEL CAMPIONE PER ANALISI MICROBIOLOGICHE

Alla luce di tutto questo, quando si analizza il problema del confezionamento, spedizione, trasporto, accettazione del campione per analisi microbiologiche, è evidente che si deve: 
- Garantire la sicurezza del personale

- Impedire la dispersione di agenti infettanti o potenzialmente infettanti nell'ambiente

- Fare in modo che il materiale giunga a destinazione nei tempi e nelle condizioni ottimali per poter essere analizzato

- Garantire la sicurezza del personale di laboratorio che riceve i campioni

- Garantire l'attendibilità dei risultati degli esami

Il problema è stato ampiamente discusso ed affrontato a livello internazionale ed il Comitato di esperti sul trasporto di merci pericolose delle nazioni unite (UN Committee of experts on the transport of dangerous goods) ha emanato delle raccomandazioni che sono state recepite nei regolamenti di varie organizzazioni che si occupano di trasporti: ICAO (International Civil Aviation Organization), IATA (International Air Transport Association Dangerous Goods Regulations), ADR (European Agreement of International Carriage of Dangerous Goods), UPU (Unione Postale Universale). L'Organizzazione Mondiale della Sanità (OMS) e il Center for Diseases Control (CDC), sulla base di tali raccomandazioni, hanno emanato linee guida concernenti la sicurezza del trasporto di materiali infetti. Il Ministero della Salute ha emanato la circolare ${ }^{\circ} 16$ del 20 luglio 1994 riportante le indicazioni per la "Spedizione di materiali biologici deperibili e/o potenzialmente infetti" e successivamente la Circolare $\mathrm{n}^{\circ} 3$ dell' 8 maggio 2003 su "Raccomandazioni per la sicurezza del trasporto di materiali infettivi e campioni diagnostici". Quest'ultima è un aggiornamento ed integrazione della Circolare $\mathrm{n}^{\circ} 16$ sulla base delle Raccomandazioni sopra citate e delle linee guida diffuse dall'OMS e riporta tutte le indicazioni a cui ci si deve attenere (8).

\section{DEFINIZIONI}

Prodotti biologici: materiali biologici finiti ad uso umano e veterinario, compresi sieri e vaccini, prodotti secondo requisiti sanciti dalla normativa vigente e trasportati dietro approvazione o permesso dell'Autorità Sanitaria; prodotti biologici finiti, trasportati prima di aver ottenuto il permesso, per scopi di studio e di ricerca umana o veterinaria; prodotti destinati al trattamento sperimentale di animali, preparati in ottemperanza alle normative vigenti.

Campioni diagnostici: tutti i materiali di origine umana o animale inclusi escreti, sangue e suoi componenti, tessuti e fluidi tissutali, raccolti a scopo diagnostico. Sono esclusi gli animali vivi infetti ed i campioni diagnostici raccolti durante un'epidemia di malattia grave e di natura scono- sciuta che, invece, devono essere trattati come sostanze infettive.

Secondo l'OMS i campioni normalmente utilizzati nella pratica clinica, a scopo diagnostico e di ricerca, sono considerati "pericolo trascurabile" per la sanità pubblica, a meno che non siano raccolti in fase di epidemia acuta da agente sconosciuto: in tal caso vanno considerati "sostanze infettive". Di conseguenza un campione di sangue per indagini relative ad HIV, un campione di escreato per ricerca Mycobacterium tuberculosis, un aspirato nasofaringeo per ricerca Coronovirus della SARS, vanno considerati "campioni diagnostici", mentre una coltura cellulare infettata da HIV o dal Coronavirus della SARS o una coltura di Mycobacterium tuberculosis vanno considerate "sostanze infettive"

Sostanze infettive: sono i materiali contenenti microorganismi vivi, quali batteri, virus, rickettsie, parassiti, funghi o tossine da essi prodotto, noti o ritenuti causa probabile di malattia infettiva nell'uomo o negli animali. Comprendono:

- colture che contengono o che potrebbero contenere agenti infettivi

- campioni umani o animali che contengono un agente infettivo. Secondo l'OMS l'agente infettivo deve essere presente in quantità sufficiente, a causare infezione quale conseguenza di esposizione in seguito ad evento accidentale durante il trasporto

- campioni provenienti da pazienti con malattia grave da causa sconosciuta

- campioni non appartenenti alle categorie sopra citate, ma definiti come infettivi da persone qualificate (medici, operatori sanitari, ricercatori)

\section{Confezionamento di sostanze infettive}

Tutte le sostanze infettive, identificate secondo le definizioni citate vanno confezionate secondo le norme UN, classe 6.2 corrispondenti alle istruzioni IATA PI 602 (garanzia che gli imballaggi hanno superato, senza danno, test di performance quali la caduta da un'altezza di $9 \mathrm{~m}$ e prove di resistenza alla perforazione):

contenitore primario: deve essere di materiale impermeabile, a tenuta stagna, con chiusura ermetica, etichettato ed avvolto in materiale assorbente non particolato, quale carta bibula o cotone idrofilo, presente in quantità sufficiente ad assorbire tutto il liquido fuoriuscito in caso di rottura del recipiente

contenitore secondario: deve essere di materiale resistente, impermeabile, a tenuta stagna, adatto a contenere e proteggere il contenitore primario. Può contenere anche più contenitori primari purchè adeguatamente collocati e singolarmente avvolti in materiale assorbente. All'esterno del 
contenitore secondario devono essere incluse le schede riguardanti i dati identificativi e descrittivi del contenuto ed i dati riguardanti il destinatario e il mittente

contenitore esterno: in esso va collocato il contenitore secondario per evitare danni causati da fattori esterni quali agenti fisici o acqua. Può essere di cartone rigido, plastica, legno o altri materiali resistenti ad urti e intemperie (figura I)

Figura I. Confezionamento sostanze infettive

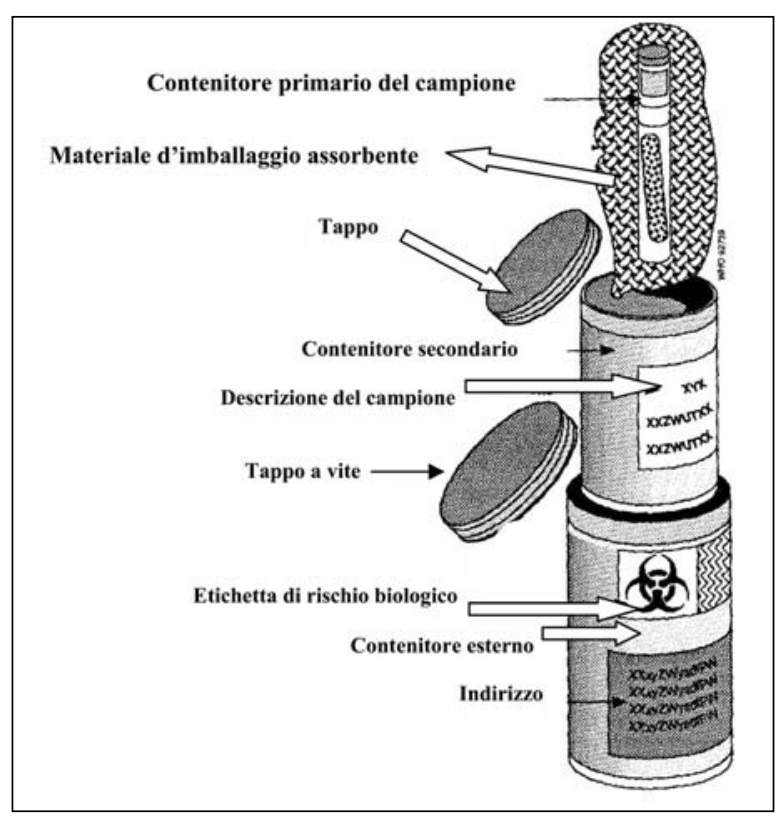

\section{Spedizione di sostanze infettive}

Limiti di quantità:

- il volume totale del materiale da spedire non può superare i $50 \mathrm{ml}-50 \mathrm{gr}$ se il trasporto avviene con aerei per passeggeri (WHO)

- il limite per il trasporto tramite cargo o altri trasportatori è 4l-4Kg totali (confezioni singole da $500 \mathrm{ml}-500 \mathrm{gr}$ ) (WHO)

- nel caso il contenitore primario superi i $50 \mathrm{ml}$ è necessario aggiungere far il recipiente secondario ed il recipiente esterno, una ulteriore quantità di materiale assorbente (WHO)

Secondo la Circolare $n^{\circ} 3$ dell' 8 maggio 2003 il volume totale non deve mai superare i $500 \mathrm{ml}$.

Esiste anche una Norma Europea, recepita in Italia (UNI EN 829, 1996) relativa alle conformità degli imballaggi

Sul contenitore esterno devono essere presenti (Figura II):

- Etichetta internazionale per le sostanze infettive

- Marchio specifico UN di confezionamento Dimensioni minime di marchio ed etichetta: 100 x $100 \mathrm{~mm}$. Per i pacchi più piccoli le dimensioni dell'etichetta devono essere di 50 x $50 \mathrm{~mm}$

- Etichetta di orientamento (dimensioni minime 74 x $105 \mathrm{~mm}$ )

- Scheda riportante nome, indirizzo, numero di telefono del ricevente e del mittente, nome UN

Figura II. Etichettatura confezione sostanze infettive

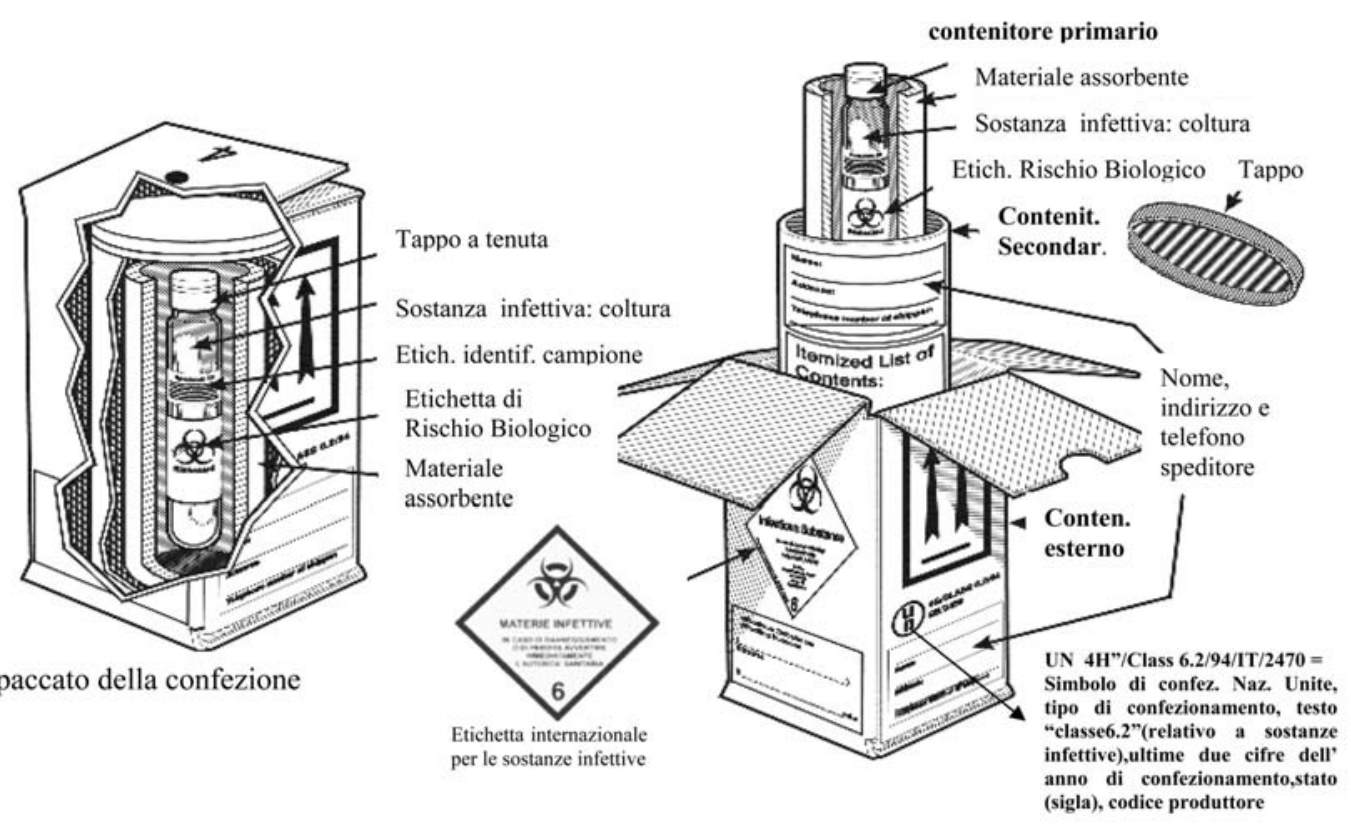

Dimensioni minime etichetta e marchio : $100 \times 100 \mathbf{~ m m}$.

Per pacchi piccoli, dimensioni etichetta : $50 \times 50 \mathbf{~ m m}$.

Dimensioni minime etichetta di orientamento (da usarsi se il

volume supera i $50 \mathrm{ml}$ ): $74 \times 105 \mathrm{~mm}$ 
(sostanza infettiva per l'uomo o per gli animali) seguito dal nome scientifico, numero UN della sostanza (UN 2814=di derivazione umana, UN $2900=$ di derivazione animale), temperatura di conservazione della sostanza.

\section{Trasporto}

Richiede i seguenti documenti, forniti dal corriere ed applicati sul contenitore esterno:

- dichiarazione di merce pericolosa da parte del corriere

- lista di imballaggio che includa l'indirizzo del destinatario, il numero di confezioni, la descrizione del contenuto, il peso, il valore (indicare se si tratta di prodotti senza valore commerciale poiché forniti a titolo gratuito)

- ricevuta aerea se il trasporto avviene per via aerea

- ricevuta di spedizione relativa ad altre modalità di trasporto, ove applicabile

- permesso di import ed export

\section{Confezionamento di campioni diagnostici}

I campioni diagnostici vanno confezionati secondo le norme UN corrispondenti alle istruzioni IATA PI 650 (Figura III):

Devono essere utilizzati tre contenitori come riportato per le sostanze infettive. Sul contenitore esterno va collocata un'etichetta riportante nome, indirizzo, numero di telefono del destinatario e del mittente e la dichiarazione "Campione diagnostico confezionato in conformità alle norma IATA PI650”.

\section{Spedizione di campioni diagnostici}

Limiti di quantità:

- il contenitore primario può contenere una quantità di materiale fino a $500 \mathrm{ml}$ e raggiungere un volume totale, nell'intera confezione, non superiore a 41 .

\section{Trasporto}

- Richiede i seguenti documenti, forniti dal corriere ed applicati sul contenitore esterno:

- lista di imballaggio che includa l'indirizzo del destinatario, il numero di confezioni, la descrizione del contenuto, il peso, il valore (indicare se si tratta di prodotti senza valore commerciale poiché forniti a titolo gratuito)

- ricevuta aerea se il trasporto avviene per via aerea

- ricevuta di spedizione relativa ad altre modalità di trasporto, ove applicabile

- permesso di import ed export se richiesto

- temperatura di conservazione del materiale

\section{TRASPORTO LOCALE}

Per trasporto locale si intende il trasporto di un campione da un reparto ospedaliero o da una struttura periferica ad un laboratorio o da un labo-

Figura III. Confezionamento etichettatura dei campioni diagnostici

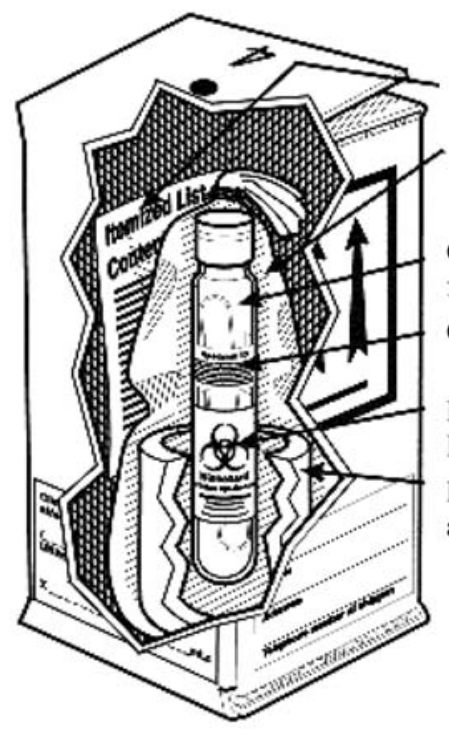

Spaccato delle confezioni

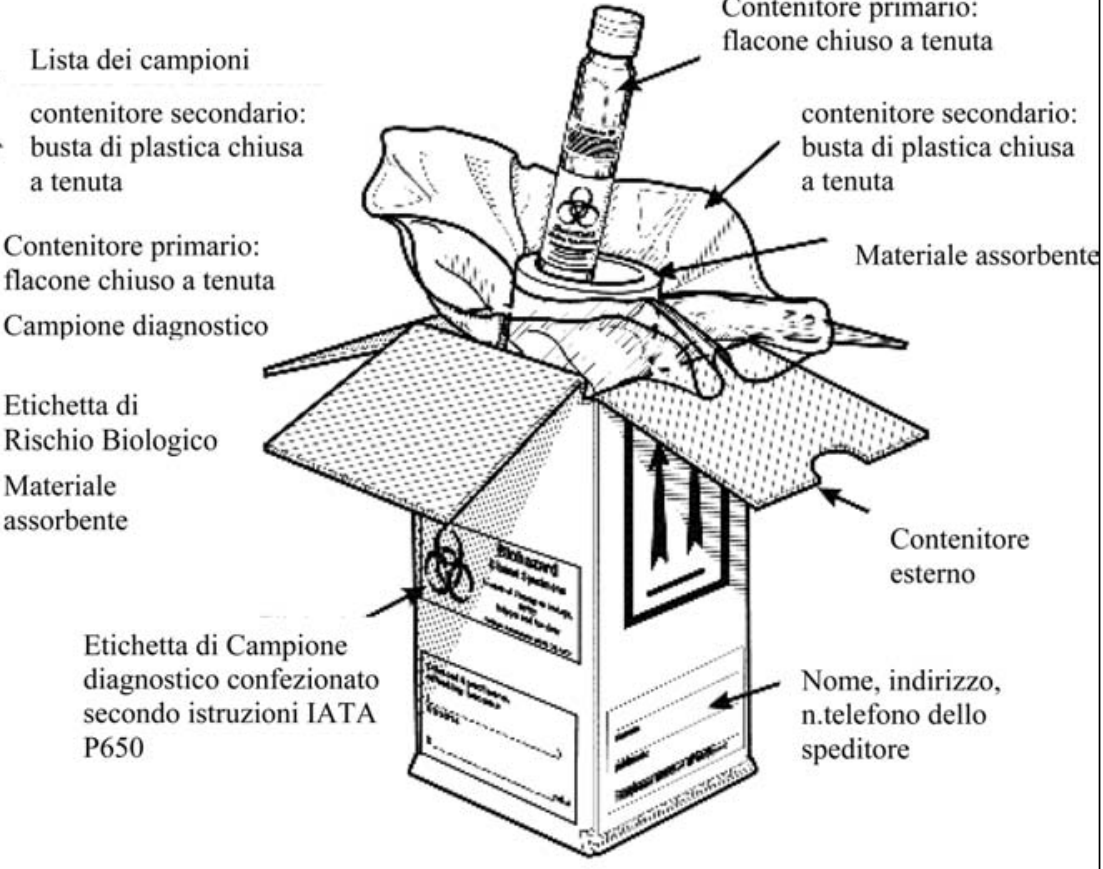

Contenitore primario:

contenitore secondario: busta di plastica chiusa a tenuta 
ratorio ad un altro, ovvero da una struttura ospedaliera ad un centro diagnostico esterno.

Vanno applicati gli stessi principi di sicurezza richiesti per le altre modalità di trasporto.

- Contenitore primario: deve essere impermeabile ed a tenuta stagna. Se il contenitore è una piastra, va opportunamente sigillata. Se il contenitore è una provetta, deve essere chiusa e collocata in una rastrelliera che la mantenga in posizione verticale.

- Contenitore secondario: i contenitori primari e le rastrelliere vanno collocate in contenitori secondari robusti e a tenuta stagna, di plastica o di metallo ed ognuno va etichettato in relazione al contenuto ed accompagnato dalle schede con i dati del campione. A nostro parere sarebbe opportuno dotare il contenitore secondario con materiale assorbente non particolato per tamponare eventuali fuoriuscite accidentali di campione.

- Quando per il trasporto del campione è previsto l'uso di veicoli, il contenitore secondario va sistemato in modo fermo e sicuro nel veicolo stesso e, a bordo, deve essere presente un kit contenente materiale assorbente, disinfettante a base di cloro, contenitore per rifiuti e guanti da lavoro resistenti e riutilizzabili.

Quando il trasporto è su strada, è preferibile, a nostro parere, utilizzare anche il terzo contenitore per una maggior tutela della salute del trasportatore in caso di incidente automobilistico.

Per un coordinamento ottimale del trasporto locale è di primaria importanza l'organizzazione accurata e precisa dei percorsi, una formazione adeguata del personale, la stesura di linee guida aziendali che regolino tutto il sistema del trasporto.

\section{Situazioni particolari: richiesta di refrigeranti per la spedizione}

Se la spedizione richiede ghiaccio o ghiaccio secco, questo va posto all'esterno del contenitore secondario. Il ghiaccio va sistemato in contenitore a tenuta stagna, impermeabile, il ghiaccio secco va sistemato in contenitore che permetta il rilascio di $\mathrm{CO}_{2}$. L'utilizzo di ghiaccio secco va indicato nella dichiarazione di merce pericolosa fatta dallo speditore e sul pacco deve essere presente l'etichetta apposita per il ghiaccio secco:

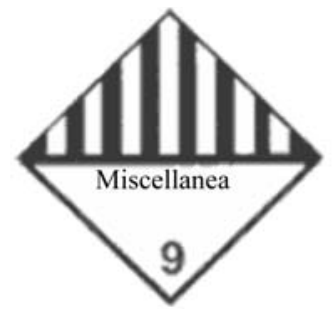

Nel caso sia invece necessario l'azoto liquido vanno presi accordi specifici con il trasportatore ed al momento del confezionamento è necessario assicurarsi che i contenitori sia adatti per l'azoto liquido ed apporre sul contenitore esterno l'apposita etichetta per l'azoto liquido:

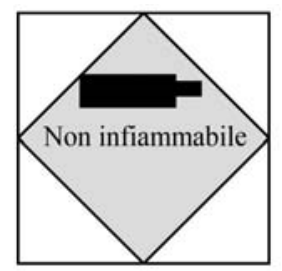

Per quanto riguarda le responsabilità specifiche del mittente, corriere e destinatario si rimanda alla Circolare $\mathrm{n}^{\circ} 3,8$ maggio 2003.

\section{Malattie infettivi attribuibili ad incidenti di trasporto: situazione attuale}

Fino ad ora non sono stati segnalati casi di malattie infettive attribuibili alla dispersione di materiale dovuta ad incidenti di trasporto, nonostante siano occorsi numerosi incidenti a causa di confezionamento non appropriato dei campioni

\section{Compiti del tecnico di laboratorio}

Di fronte a tutte queste problematiche, essendo il tecnico uno degli anelli principali nella catena della gestione del campione biologico viene spontaneo chiedersi che cosa deve fare. Fondamentale è indubbiamente:

- sottoporsi alle vaccinazioni quali quelle dell'epatite $\mathrm{B}$

- conoscere e seguire le norme di precauzione universale

- conoscere, saper utilizzare e seguire la manutenzione dei Dispositivi di Protezione Collettiva o DPC (sistemi di ventilazione, cappe di biosicurezza ecc.)

- controllare costantemente se i campioni arrivano correttamente confezionati e nei tempi previsti

- compilare la scheda di non conformità alle norma di sicurezza (Figura IV)

- far riferimento al responsabile del rischio biologico della propria struttura per risolvere i problemi che man mano si presentano

\section{Norme di precauzione universali}

Le precauzioni universali devono essere adottate routinariamente per prevenire l'esposizione cutanea, mucosa o parentereale nei casi in cui si preveda (anche solo accidentalmente) il contatto con sangue o altri liquidi biologici provenienti da qualsiasi paziente. Consistono nell'adozione di misure di barriera o Dispositivi di Protezione Individuale (DPI) quali guanti, camici, maschere, occhiali, schermi facciali protettivi, grembiuli, calzari ecc..., nel corretto uso e smaltimento di aghi e taglienti, nel corretto lavaggio delle mani, nell'immediata 
Figura IV. Esempio di scheda per il rilevamento delle non conformità relative al confezionamento e trasporto dei campioni biologici

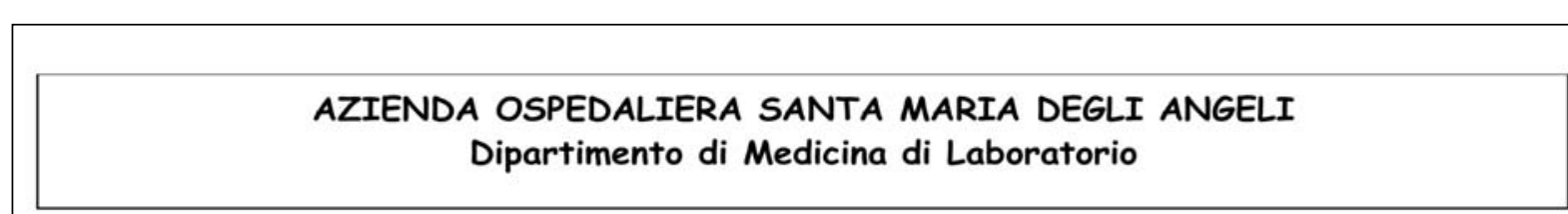

\section{SEGNALAZIONE DI NON CONFORMITA' RELATIVA AL CONFEZIONAMENTO E TRASPORTO CAMPIONI BIOLOGICI}

\begin{tabular}{|lr|l|l|l|}
\hline \multicolumn{5}{|c|}{ STRUTTURA OPERATIVA CHE RILEVA LA NON CONFORMITA' } \\
\hline Anatomia Patologica & $\square$ & Citogenetica & $\begin{array}{l}\text { Immunologia Clinica e } \\
\text { Virologia }\end{array}$ & Microbiologia Clinica e \\
& $\square$ & & & Tera pia antibiotica \\
\hline Patologia Clinica & $\square$ & Servizio & $\square$ \\
& Immunotrasfusionale $\square$ & & \\
\hline
\end{tabular}

\begin{tabular}{|c|c|c|c|}
\hline Data & $\begin{array}{l}n^{\circ} \text { e tipo di } \\
\text { campione }\end{array}$ & Provenienza & Descrizione non conformità \\
\hline & & & $\begin{array}{l}\square \text { Provetta o contenitore imbrattato } \\
\square \text { Tappo non chiuso correttamente } \\
\square \text { Contenitori per il trasporto non adeguati } \\
\text { Altro } \\
\text { Soluzione del problema }\end{array}$ \\
\hline & & & Rilevatore \\
\hline & & & $\begin{array}{l}\square \text { Provetta o contenitore imbrattato } \\
\square \text { Tappo non chiuso correttamente } \\
\square \text { Contenitori per il trasporto non adeguati } \\
\text { Altro } \\
\text { Soluzione del problema }\end{array}$ \\
\hline & & & Rilevatore \\
\hline & & & $\begin{array}{l}\square \text { Provetta o contenitore imbrattato } \\
\square \text { Tappo non chiuso correttamente } \\
\square \text { Contenitori per il trasporto non adeguati } \\
\text { Altro } \\
\text { Soluzione del problema }\end{array}$ \\
\hline & & & Rilevatore \\
\hline & & & $\begin{array}{l}\square \text { Provetta o contenitore imbrattato } \\
\square \text { Tappo non chiuso correttamente } \\
\square \text { Contenitori per il trasporto non adeguati } \\
\text { Altro } \\
\text { Soluzione del problema }\end{array}$ \\
\hline & & & Rilevatore \\
\hline
\end{tabular}

decontaminazione delle superfici sporche di materiali biologici potenzialmente infetti.

Le maschere, gli occhiali, gli schermi facciali protettivi vanno utilizzati ogniqualvolta vi sia la possibilità di formazione di schizzi di sangue o altri campioni biologici che possono contaminare le mucose della bocca e degli occhi.

Da tener presente che gli aghi non devono mai essere reincappucciati, piegati, rimossi dalle siringhe monouso o dalle loro custodie o altrimenti 
manipolati con le mani. Aghi e taglienti devono essere eliminati negli appositi contenitori a parete rigida, facendo attenzione ad operare con la massima cautela in modo che al momento dell'introduzione nel contenitore non vi sia rischio di puntura o tagli accidentali.

Per decontaminare immediatamente superfici sporche di materiali biologici potenzialmente infetti si opera come segue:

- indossare guanti di gomma per le pulizie

- rimuovere il materiale visibile con materiale assorbente (es. salviette di carta)

- decontaminare l'area con una soluzione di cloroderivato (5.000-10.000 ppm) utilizzando uno straccio monouso e lasciando agire il disinfettante per almeno 10 minuti

- lavare l'area con un detergente e, dopo asciugatura, disinfettare con il cloroderivato

- risciacquare

- eliminare il materiale utilizzato negli appositi contenitori per rifiuti ospedalieri da mandare all'inceneritore.

- In caso di versamento abbondante di materiale biologico, dopo aver indossato i guanti di gomma, coprire la superficie interessata con salviette di carta o altro materiale assorbente e versare la soluzione di cloroderivato, lasciar agire per 20 minuti, asportare il liquido versato con materiale assorbente ed eliminarlo negli appositi contenitori. Procedere poi come sopra partendo dal lavaggio con detergente.

\section{CONCLUSIONI}

Le organizzazioni internazionali, la legislazione italiana con il D.L. 626/94 e successive modifiche hanno portato alla ribalta il problema della tutela della salute dei lavoratori in maniera innovativa ed attenta, stabilendo obblighi precisi sia per il datore di lavoro che per il lavoratore: oltre ai mezzi tecnici che l'industria fornisce per il contenimento ci sono ora anche i mezzi legali per provvedere al meglio a proteggere noi stessi. Sono anche ormai messe a punto, come visto, tutte le norme comportamentali per quanto riguarda la gestione del campione biologico, dal prelievo fino all'arrivo in laboratorio.

Sta ora a tutti seguire in maniera costante e precisa le indicazioni, segnalare i problemi che quotidianamente possono presentarsi e le eventuali carenze organizzative, facendo attenzione a non calare mai la guardia nei confronti di queste problematiche.

Si potranno così evitare esposizioni pericolose e nel contempo garantire un percorso corretto del campione che non potrà che avere impatto positivo sulla qualità del risultato finale.

\section{BIBLIOGRAFIA}

1. Abdala N, Stephens PC, Griffith BP, Heimer R. Survival of HIV-1 in syringes. J Acquir Immune Defic Syndr Hum Retrovirol 1999 Jan 1; 20 (1): 73-80.

2. Abdala N, Reyes R, Carney JM, Heimer R. Survival of HIV-1 in syringes: effects of temperature during storage. Subst Use Misuse 2000 Aug; 35 (10): 1369-83.

3. Aitken C, Jeffries DJ. Nosocomial Spread of Viral Disease. Clin Microbiol Rev 2001 July; 14 (3): 528-46.

4. Archuleta RJ, Mullens P, Primm TP. The relationship of temperature to desiccation and starvation tolerance of the Mycobacterium avium complex. Arch Microbiol 2002 Oct; 178 (4): 311-4.

5. Beltrami EM, Williams IT, Shapiro CN, Chamberland ME. Risk and Management of Blood-Borne Infections in Health Care Workers. Clin Microbiol Rev 2000; 13 (3): 385-407.

6. Bolyard EA, Tablan OC, Williams WW, Pearson ML, Shapiro CN, Deitchman SD, The Hospital Infection Control Practices Advisory Committee. Guideline for infection control in health care personnel, 1998. CDC Personnel Health Guideline. AJIC June 1998: 289-354.

7. Cardoso CL, Giacomelli LR, Helbel C, Sant'Ana JJ, Martins FM, Barreto AM. Survival of tubercle bacilli in heat-fixed and stained sputum smears. Mem Inst Oswaldo Cruz 2001 Feb; 96 (2): 277-80.

8. Circolare $\mathrm{n}^{\circ}$ 3, 8 maggio 2003: Raccomandazioni per la sicurezza del trasporto di materiali infettivi e di campioni diagnostici. Ministero della Salute. Direzione Generale della Prevenzione. Ufficio III. Malattie Infettive e profilassi internazionale-OEN 400.3/113.2/82/2662.

9. Do AN, Ciesielski CA, Metler RP, Hammett TA, Fleming PL. Occupationally acquired human immunodeficiency virus (HIV) infection: national case surveillance data during 20 years of the HIV epidemic in the United States. Infect Control Hosp Epidemiol 2003 Feb; 24 (2): 86-96.

10. Duan SM, Zhao XS, Wen RF, et al. SARS Research Team. Stability of SARS coronavirus in human specimens and environment and its sensitivity to heating and UV irradiation. Biomed Environ Sci 2003 Sep;16 (3): 246-55.

11. Grist NR, Emslie JA. Infections in British clinical laboratories, 1988-1989. J Clin Pathol 1991 Aug; 44 (8): 667-9.

12. Guidelines for the safe transport of infectious substances and diagnostic specimens. World Health Organization, Division of Emerging and other Communicable Diseases Surveillance and control. WHO/EMC/97.3, Geneva, 1997

13. Heimer R, Abdala N. Viability of HIV-1 in syringes: implications for interventions among injection drug users. AIDS Read 2000 Jul; 10 (7): 410-7.

14. Hirai Y. Survival of bacteria under dry conditions; from a viewpoint of nosocomial infection. J Hosp Infect 1991 Nov; 19 (3): 191-200.

15. Pike RM. Laboratory-associated infections: summary and analysis of 3921 cases. Helth Lab Sci 1976 Apr; 13 (2): 105-14.

16. Sewell DJ. Laboratory-Associated Infections in Biosafety. Clin Microbiol Rev 1995; 8 (3): 389-405.

17. Smith SM, Eng RH, Padberg FT. Survival of nosocomial pathogenic bacteria at ambient temperature. J Med 1996; 27(5-6): 293-302.

18. Walker D, Campbell D. A survey of infections in United Kingdom laboratories, 1994-1995. J Clin Pathol 1999 Jun; 52 (6): 415-8. 